\title{
ASYMPTOTIC BEHAVIOUR OF NON-AUTONOMOUS DISSIPATIVE SYSTEMS IN HILBERT SPACE
}

\author{
SONG GUOZHU
}

(Received 20 May 1994; revised 19 May 1995)

Communicated by E. N. Dancer

\begin{abstract}
In this paper we discuss the asymptotic behaviour, as $t \rightarrow \infty$, of the integral solution $u(t)$ of the nonlinear evolution equation $u^{\prime}(t) \in A(t) u(t)+g(t), t \geq s, u(s)=x_{0} \in \overline{D(A(s))}$, where $\{A(t)\}_{t \geq 0}$ is a family of $m$-dissipative operators in a Hilbert space $H$, and $g \in L_{l o c}(0, \infty ; H)$. We give some sufficient conditions and some sufficient and necessary conditions to ensure that $\sigma(t)=t^{-1} \int_{s}^{s+t} u(\theta) d \theta$ and $u(t)$ are weakly convergent.
\end{abstract}

1991 Mathematics subject classification (Amer. Math. Soc.): 47B44, 34G20.

Keywords and phrases: Non-linear evolution equation, dissipative operator, integral solution, asymptotic behaviour.

\section{Introduction and preliminaries}

Let $H$ be a real Hilbert space with inner product (,) and norm |,|. We consider the non-linear evolution equation

$$
\left\{\begin{array}{l}
u^{\prime}(t) \in A(t) u(t)+g(t), \quad t \geq s \\
u(s)=x_{0}
\end{array}\right.
$$

where $\{A(t)\}_{t \geq 0}$ is a family of $m$-dissipative operators in $H, x_{0} \in \overline{D(A(s))}$ and $g \in L_{l o c}(0, \infty ; H)$. Our objective is to study the asymptotic behaviour, as $t \rightarrow \infty$, of the integral solution $u(t)$ of $(1.1)$. In $[6,7,9]$ the weak convergence of the autonomous dissipative system

$$
\left\{\begin{array}{l}
u^{\prime}(t) \in A u(t) \\
u(0)=x_{0}
\end{array}\right.
$$

(C) 1997 Australian Mathematical Society 0263-6115/97 \$A2.00+0.00 
where $A$ is an $m$-dissipative operator in $H, x_{0} \in \overline{D(A)}$, has been studied. In [4, 10] Morosanu and Rouhani discussed the weak convergence of the quasi-autonomous dissipative system

$$
\left\{\begin{array}{l}
u^{\prime}(t) \in A u(t)+g(t) \\
u(0)=x_{0}
\end{array}\right.
$$

where $g \in L(0, \infty ; H)$ (or more generally, $g-g_{\infty} \in L(0, \infty ; H)$ for some $g_{\infty} \in H$ ).

Throughout this paper we assume that $A(t)$ satisfies the following conditions:

$\left(H_{1}\right)$ : there exists a continuous function $f: R_{+} \rightarrow H$ and a bounded (on bounded subsets) function $L: R_{+} \rightarrow R_{+}$such that

$$
\left(y_{1}-y_{2}, x_{1}-x_{2}\right) \leq|f(t)-f(s)| \cdot\left|x_{1}-x_{2}\right| \cdot L\left(\left|x_{2}\right|\right)
$$

for all $0 \leq s \leq t,\left[x_{1}, y_{1}\right] \in A(t),\left[x_{2}, y_{2}\right] \in A(s)$.

$\left(H_{2}\right)$ : If $t_{n} \uparrow t$ in $[s,+\infty], x_{n} \in D\left(A\left(t_{n}\right)\right), x_{n} \rightarrow x$ in $H$, then $x \in \overline{D(A(t))}$.

DEFINITION 1.1. If $u(t)$ is continuous on $[s, \infty), u(s)=x_{0}, u(t) \in \overline{D(A(t))}$ for $t \in[s, \infty)$ and satisfies the inequality

$$
|u(\bar{t})-x| \leq|u(t)-x|+\int_{t}^{\bar{t}}\left(\langle y+g(\theta), u(\theta)-x\rangle_{+}+c \mid f(\theta-f(r) \mid) d \theta\right.
$$

for all $s \leq t \leq \bar{t}, r \geq s$ and $[x, y] \in A(r)$. Then $u(t)$ is called an integral solution to (1.1). Here $c=L(|x|),\langle y, x\rangle_{+}=\lim _{h \downarrow 0}(|x+h y|-|x|) / h$ and $(y, x)=|x|\langle y, x\rangle_{+}$.

Clearly, a strong solution $u(t)$ to (1.1) is automatically an integral solution to (1.1), and by [5] the problem (1.1) has a unique integral solution under our hypotheses, and the inequality (1.3) is equivalent to

$$
\begin{aligned}
\frac{1}{2}\left(|u(\bar{t})-x|^{2}-|u(t)-x|^{2}\right) \leq & \int_{t}^{\bar{t}}(g(\theta)+y, u(\theta)-x) d \theta \\
& +L(|x|) \int_{t}^{\bar{t}}|u(\theta)-x| \cdot|f(\theta)-f(r)| d \theta
\end{aligned}
$$

for all

$$
s \leq t \leq \bar{t}, \quad r \geq s, \quad[x, y] \in A(r) .
$$




\section{Weak convergence of the integral solution}

LEMMA 2.1. Suppose $F$ is a non-empty closed convex set in $H, P_{F}$ is a projection on $F$. Then

(2.1) $\left(x-P_{F} x, z-P_{F} x\right) \leq 0, \quad \forall z \in F, x \in H$.

(2.2) $\left|P_{F} x-P_{F} y\right| \leq|x-y| \quad \forall x, y \in H$.

(2.3) $\left|P_{F} x-z\right|^{2} \leq|x-z|^{2}-\left|P_{F} x-x\right|^{2}, \quad \forall x \in H, z \in F$.

Since Lemma 2.1 is well known, its proof will be omitted.

LEMMA 2.2. Suppose $u(t)$ is an integral solution to (1.1). If there are $r_{0} \geq s$ and $g_{\infty} \in H$ such that $f-f\left(r_{0}\right) \in L(0, \infty ; H)$ and $g-g_{\infty} \in L(0, \infty ; H)$, then $u(t)$ is bounded on $[s, \infty)$ if and only if $A^{-1}\left(r_{0}\right)\left(-g_{\infty}\right) \neq \emptyset$.

PROOF. Firstly, we suppose that $u(t)$ is bounded on $[s, \infty)$. Since $u(t)$ is an integral solution of (1.1), then for all $t \geq s \geq 0$ and $[x, y] \in A\left(r_{0}\right)$ we have

$$
\begin{aligned}
\left.\left.\frac{1}{2}(\mid u(t)-x)\right|^{2}-\left|u_{0}-x\right|^{2}\right) \leq & \int_{s}^{t}(g(\theta)+y, u(\theta)-x) d \theta \\
& +L(|x|) \int_{s}^{t}|u(\theta)-x| \cdot\left|f(\theta)-f\left(r_{0}\right)\right| d \theta .
\end{aligned}
$$

Dividing by $t-s>0$, we obtain

$$
\begin{array}{r}
\frac{1}{2(t-s)}\left(|u(t)-x|^{2}-\left|u_{0}-x\right|^{2}\right) \leq \frac{1}{t-s} \int_{s}^{t}\left(g(\theta)-g_{\infty}, u(\theta)-x\right) d \theta \\
\quad+\left(y+g_{\infty}, \sigma(t)-x\right)+\frac{L(|x|)}{t-s} \int_{s}^{t}|u(\theta)-x| \cdot\left|f(\theta)-f\left(r_{0}\right)\right| d \theta
\end{array}
$$

for all $t>s \geq 0,[x, y] \in A\left(r_{0}\right)$, where $\sigma(t)=(t-s)^{-1} \int_{s}^{t} u(\theta) d \theta$ is bounded on $[s, \infty)$. Therefore there exists a sequence $t_{n} \rightarrow \infty$ such that $\sigma\left(t_{n}\right)$ converges weakly to $p \in H$. If we take $t=t_{n}$ in (2.5) and let $n \rightarrow \infty$, then

$$
\left(y+g_{\infty}, x-p\right) \leq 0 \text { for all }[x, y] \in A\left(r_{0}\right) .
$$

The maximality of $A\left(r_{0}\right)$ implies that $\left[p,-g_{\infty}\right] \in A\left(r_{0}\right)$, that is, $A^{-1}\left(r_{0}\right)\left(-g_{\infty}\right)$ is non-empty.

Conversely, if $A^{-1}\left(r_{0}\right)\left(-g_{\infty}\right) \neq \emptyset$, then there exists an element $x \in D\left(A\left(r_{0}\right)\right)$ such that $-g_{\infty} \in A\left(r_{0}\right) x$. We take $y=-g_{\infty}$ in (2.4) and by a variant of Gronwall's Lemma (see $[2$, p. 157]) we deduce that $u(t)$ is bounded on $[s, \infty)$. The proof is complete. 
THEOREM 2.3. Suppose $u(t)$ is an integral solution to (1.1). If there are $f_{\infty}$, $g_{\infty} \in H$ and $\tau_{n} \rightarrow \infty$ such that $f\left(\tau_{n}\right)=f_{\infty}(n \in N), F=\bigcap_{n=1}^{\infty} A^{-1}\left(\tau_{n}\right)\left(-g_{\infty}\right) \neq \emptyset$, then there is $p \in F$ such that

$$
P_{F} u(t) \stackrel{s}{\rightarrow} p \quad \text { and } \quad \sigma(t) \stackrel{w}{\rightarrow} p(t \rightarrow \infty)
$$

Proof. We may assume $g_{\infty}=0, F=\bigcap_{n=1}^{\infty} A^{-1}\left(\tau_{n}\right)(0)$ (without loss of generality). Since $A^{-1}(t)$ is maximal dissipative, then $F$ is a closed convex subset in $H$. Take $x \in F, r=\tau_{n}$. By the 'if' part of Lemma 2.2, $u(t)$ is bounded on $[s, \infty)$ and for all $x \in F, \bar{t} \geq t \geq s \geq 0$

$$
|u(\bar{t})-x|-|u(t)-x| \leq \int_{s}^{\bar{t}}\left(|g(\theta)|+L(|x|)\left|f(\theta)-f_{\infty}\right|\right) d \theta .
$$

Hence, for every $x \in F$, the function $t \rightarrow|u(t)-x|-\int_{0}^{t}\left(g(\theta)-L(|x|)\left|f(\theta)-f_{\infty}\right|\right) d \theta$ is non-increasing and bounded on $[s, \infty)$. Since $g, f-f_{\infty} \in L(0, \infty ; H)$ we conclude that there exists a limit

$$
\lim _{t \rightarrow \infty}|u(t)-x|=\alpha(x) \quad \text { for every } x \in F .
$$

We set $v(t)=P_{F} u(t)$. According to Lemma 2.1 (ii), $v(t)$ is bounded on [s, $\infty$ ). Let $C_{1}=\sup _{t \geq s} L(|v(t)|)$; for fixed $t \geq s$ we denote $y_{t}(h)=u(t+h), h \geq 0$. Then $y_{t}(h)$ is an integral solution of the following equation:

$$
\left\{\begin{array}{l}
\frac{d y_{t}(h)}{d h} \in A(t+h) y_{t}(h)+g(t+h) \\
y_{t}(0)=u(t) .
\end{array}\right.
$$

By the same argument above we obtain the function

$$
h \rightarrow\left|y_{t}(h)-v(t)\right|-\int_{0}^{h}\left(|g(\theta+t)|+C_{1}\left|f(\theta+t)-f_{\infty}\right|\right) d \theta
$$

is non-increasing. Hence $\forall t \geq s, h \geq 0$,

$$
|u(t+h)-v(t)|-\int_{t}^{t+h}\left(|g(\theta)|+C_{1}\left|f(\theta)-f_{\infty}\right|\right) d \theta \leq|u(t)-v(t)| .
$$

This implies that for all $t \geq s, h \geq 0$,

$$
\begin{aligned}
\mid u(t+ & h)-v(t+h) \mid-\int_{s}^{t+h}\left(|g(\theta)|+C_{1}\left|f(\theta)-f_{\infty}\right|\right) d \theta \\
\leq & |u(t+h)-v(t)|-\int_{s}^{t}\left(|g(\theta)|+C_{1}\left|f(\theta)-f_{\infty}\right|\right) d \theta \\
& -\int_{t}^{t+h}\left(|g(\theta)|+C_{1}\left|f(\theta)-f_{\infty}\right|\right) d \theta \\
\leq & |u(t)-v(t)|-\int_{s}^{t}\left(|g(\theta)|+C_{1}\left|f(\theta)-f_{\infty}\right|\right) d \theta .
\end{aligned}
$$


Thus the function $t \rightarrow|u(t)-v(t)|-\int_{s}^{t}\left(|g(\theta)|+C_{1}\left|f(\theta)-f_{\infty}\right|\right) d \theta$ is non-increasing on $[s,+\infty)$ and there exists $\lim _{t \rightarrow \infty}|u(t)-v(t)|$.

Next, by Lemma 2.1 (iii)

$$
|v(t+h)-v(t)|^{2} \leq|u(t+h)-v(t)|^{2}-|v(t+h)-u(t+h)|^{2} .
$$

From (2.10) and (2.11) one obtains

$$
\begin{aligned}
|v(t+h)-v(t)|^{2} \leq & |u(t)-v(t)|^{2}-|u(t+h)-v(t+h)|^{2} \\
& +2|u(t)-v(t)| \cdot \int_{t}^{t+h}\left(|g(\theta)|+C_{1}\left|f(\theta)-f_{\infty}\right|\right) d \theta \\
& +\left[\int_{t}^{t+h}\left(|g(\theta)|+C_{1}\left|f(\theta)-f_{\infty}\right|\right) d \theta\right]^{2} .
\end{aligned}
$$

This implies that there exists $\lim _{t \rightarrow \infty} v(t)=p$ and $p \in F$.

Now suppose $\sigma\left(t_{k}\right) \stackrel{w}{\rightarrow} y\left(t_{k} \rightarrow \infty\right)$. By the 'only if' part of Lemma 2.2 for every $n \in \mathscr{N}$ we have $y \in F_{n}=A^{-1}\left(\tau_{n}\right)\left(-g_{\infty}\right)$; thus $y \in F$. According to Lemma 2.1 (i) we have

$$
\begin{gathered}
(u(t)-v(t), z-v(t)) \leq 0, \quad \forall z \in F, \\
\frac{1}{t_{k}} \int_{s}^{t_{k}+s}(u(\theta)-v(\theta), z-v(\theta)) d \theta \leq 0, \quad \forall z \in F .
\end{gathered}
$$

Letting $t_{k} \rightarrow \infty$ in (2.12), one obtains

$$
(y-p, z-p) \leq 0, \quad \forall z \in F .
$$

This implies that $y=p$ and $\sigma(t) \stackrel{w}{\rightarrow} p(t \rightarrow \infty)$. The proof is complete.

REMARK 2.4. If $A(t) \equiv A, s=0$ and $F=A^{-1}\left(-g_{\infty}\right) \neq \emptyset$, then from Theorem 2.3 we may obtain respectively the Ergodic Theorem of autonomous systems and quasi-autonomous dissipative systems in $[4,10,5]$.

LEMMA 2.5. Suppose $u(t)$ is an integral solution to (1.1). Then for all $T>0, h>$ $0, r \geq \tau \geq s \geq 0$ and $r+h \leq T$, we have

$$
\begin{aligned}
& |u(r+h)-u(\tau+h)| \leq|u(r)-u(t)| \\
& \quad+\int_{\tau}^{\tau+h}\left(C_{2}|f(\theta+(r-\tau))-f(\theta)|+|g(\theta+(r-\tau))-g(\theta)|\right) d \theta
\end{aligned}
$$

where $C_{2}=\sup \{L(t): 0 \leq t \leq \sup \{|u(\theta)|: s \leq \theta \leq T+(r-\tau)\}+1\}$. 
Proof. From Theorem 1 (ii) in [11] we get

$$
\begin{aligned}
\left|u\left(t+h_{1}\right)-u(t)\right| \leq\left|u\left(\tau+h_{1}\right)-u(\tau)\right| & \\
& +\int_{\tau}^{t}\left(\tilde{C}\left|f\left(\theta+h_{1}\right)-f(\theta)\right|+\left|g\left(\theta+h_{1}\right)-g(\theta)\right|\right) d \theta
\end{aligned}
$$

for all $s \leq \tau \leq t \leq T$ and $h_{1}>0$, where $\tilde{C}=\sup \left\{L(t): 0 \leq t \leq \sup \left\{\left|u\left(\theta+h_{1}\right)\right|:\right.\right.$ $s \leq \theta \leq T\}+1\}$.

For $h>0, r \geq \tau \geq s$, let $t=\tau+h$ and $r-\tau=h_{1}$ in (2.14). One obtains (2.13). The proof is complete.

THEOREM 2.6. Suppose $u(t)$ is an integral solution to (1.1). If there are $r_{0} \geq s$ and $g_{\infty} \in H$ such that $-g_{\infty} \in R\left(A\left(r_{0}\right)\right), f-f\left(r_{0}\right) \in L(0, \infty ; H)$ and $g-g_{\infty} \in$ $L(0, \infty ; H)$, then there exists $p \in A^{-1}\left(r_{0}\right)\left(-g_{\infty}\right)$ such that $w-\lim _{t \rightarrow \infty} \sigma(t)=p$.

PROOF. Firstly, by Lemma 2.2, $\sup _{t \geq s}|u(t)|=M<\infty$. We set $\epsilon_{1}(r, \tau)=$

$$
\left\{\begin{array}{l}
\int_{\tau}^{\infty} M \mid f\left(\theta+(r-\tau)-f(\theta)\left|d \theta+\int_{\tau}^{\infty}\right| g(\theta+(r-\tau))-g(\theta) \mid d \theta, \quad r \geq \tau,\right. \\
\int_{r}^{\infty} M \mid f\left(\theta+(r-\tau)-f(\theta)\left|d \theta+\int_{r}^{\infty}\right| g(\theta+(r-\tau))-g(\theta) \mid d \theta, \quad r<\tau .\right.
\end{array}\right.
$$

Then $\lim _{r, \tau \rightarrow \infty} \epsilon_{1}(r, \tau)=0$. By Lemma 2.5 and Definition 3.1 in [10] we know that the curve $(u(t))_{t \geq s}$ is almost non-expansive in $H$. Hence by Theorem 3.8 in [10] and the 'only if' part of Lemma 2.2 there exists $w-\lim _{t \rightarrow \infty} \sigma(t)=p$ and $p \in A^{-1}\left(r_{0}\right)\left(-g_{\infty}\right)$.

COROLLARY 2.7. Suppose $u(t)$ is an integral solution to (1.1). If there are $f_{\infty}, g_{\infty} \in$ $H$ and $T>0$ such that $f-f_{\infty} \in L(0, \infty ; H), g-g_{\infty} \in L(0, \infty ; H)$ and $F=$ $\bigcap_{t \geq T} A^{-1}(t)\left(-g_{\infty}\right) \neq \emptyset$, then $\sigma(t)$ is weakly convergent as $t \rightarrow \infty$.

THEOREM 2.8. Suppose $u(t)$ is an integral solution to (1.1). If there exist $r_{0} \geq s$ and $g_{\infty} \in H$ such that $f-f\left(r_{0}\right) \in L(0, \infty ; H)$ and $g-g_{\infty} \in L(0, \infty ; H)$, then there exists $w-\lim _{t \rightarrow \infty} u(t)$ if and only if $F=A^{-1}\left(r_{0}\right)\left(-g_{\infty}\right) \neq \emptyset$ and $\omega_{w}\left(x_{0}\right) \subset F$, where $\omega_{w}\left(x_{0}\right)$ is the set of weak cluster points of $\{u(t): t \geq s\}$.

PROOF. 'Only if' part: Suppose $w-\lim _{t \rightarrow \infty} u(t)=p$. This implies that $w-\lim _{t \rightarrow \infty} \sigma(t)$ $=p$. From (2.5) it follows that $p \in F$.

'If' part: Since $F \neq \emptyset$ and $\omega_{w}\left(x_{0}\right) \subset F$, according to Lemma 2.2, $\omega_{w}\left(x_{0}\right) \neq \emptyset$. Let $p, q$ be arbitrary in $\omega_{w}\left(x_{0}\right) \subset F$. We have

$$
|u(t)-p|^{2}=|u(t)-q|^{2}+2(u(t)-q, q-p)+|q-p|^{2}, \quad t \geq s .
$$


Now for all $\bar{t} \geq t \geq s, x \in F$ we have

$$
|u(\bar{t})-x|-|u(t)-x| \leq \int_{t}^{\bar{t}}\left(\left|g(\theta)-g_{\infty}\right|+L(|x|)\left|f(\theta)-f\left(r_{0}\right)\right|\right) d \theta .
$$

Thus the function

$$
t \rightarrow|u(t)-x|+\int_{0}^{t}\left(\left|g(\theta)-g_{\infty}\right|+L(|x|)\left|f(\theta)-f\left(r_{0}\right)\right|\right) d \theta
$$

is non-increasing on $[s, \infty)$ and there exists $\lim _{t \rightarrow \infty}|u(t)-x|=\alpha(x)$. Now $p, q \in F$; then from (2.15) we get

$$
\alpha^{2}(p)-\alpha^{2}(q)=|q-p|^{2}
$$

and

$$
\alpha^{2}(q)-\alpha^{2}(p)=|p-q|^{2} .
$$

Hence $p=q, \omega_{w}\left(x_{0}\right)$ contains only one element and $w$-lim $\lim _{t \rightarrow \infty} \boldsymbol{u}(t)=p$. The proof is complete.

LEMMA 2.9. Suppose $u(t)$ is an integral solution to (1.1) with $g(t) \equiv 0$ and $x_{0}=x$ and $F$ is a closed subset of $H$. If $\omega_{w}(x) \subset F$ for all $x \in D(A(s))$ then $\omega_{w}(x) \subset F$ for all $x \in \overline{D(A(s))}$.

PROOF. Let $x \in \overline{D(A(s))}$ and let $x_{n} \rightarrow x$ with $x_{n} \in D(A(s))$. If $y \in \omega_{w}(x)$ then there exists a sequence $t_{k} \rightarrow \infty$ such that $u\left(t_{k}\right)=U\left(t_{k}, s\right) \stackrel{\stackrel{w}{\rightarrow}}{\rightarrow}$, where $U(t, s)$ is an evolution operator generated by $A(t)$. For every fixed $n$ the sequence $\left|U\left(t_{k}, s\right) x_{n}\right|$ is bounded and therefore $U\left(t_{k}, s\right) x_{n}$ has a weakly convergent subsequence $U\left(t_{k_{j}}, s\right) x_{n} \stackrel{w}{\rightarrow} y_{n}$. Clearly $y_{n} \in \omega_{w}\left(x_{n}\right) \subset F$ and

$$
\left|y_{n}-y\right| \leq \lim _{j \rightarrow \infty}\left|U\left(t_{k_{j}}, s\right) x_{n}-U\left(t_{k_{j}}, s\right) x\right| \leq\left|x_{n}-x\right| .
$$

Thus $y_{n} \rightarrow y$ and $y \in F$. The proof is complete.

THEOREM 2.10. Suppose $u(t)$ is an integral solution to (1.1) with $g(t) \equiv 0$ and $x_{0}=x$, the function $f(t)$ in the condition $\left(H_{1}\right)$ is of bounded variation on $[s, T]$ and $V_{s}^{T}(f)=M_{T} \leq M_{0}<\infty$ for all $T>s$. If there exist $T_{0}>s$ and $f_{\infty} \in H$ such that $F=\bigcap_{t \geq T_{0}} A^{-1}(t)(0) \neq \emptyset, f-f_{\infty} \in L(0, \infty ; H)$ and satisfying the condition

$\left(H_{3}\right)$ : There exists $x_{0} \in F$ such that $x_{n} \stackrel{w}{\rightarrow} x, y_{n} \in A\left(t_{n}\right) x_{n}\left(t_{n} \rightarrow \infty\right)$ and $\lim _{n \rightarrow \infty}\left(y_{n}, x_{n}-x_{0}\right)=0$ imply $x \in F$. 
Then $u(t)=U(t, s) x$ is weakly convergent as $t \rightarrow \infty$.

PROOF. Since $F$ is a closed convex subset of $H$, by Lemma 2.9 it is sufficient to prove that $\omega_{w}(x) \subset F$ for every $x \in D(A(s))$. Let $x \in D(A(s))$ and $y \in \omega_{w}(x)$ be such that $u\left(t_{k}\right)=U\left(t_{k}, s\right) x \stackrel{w}{\rightarrow} y\left(t_{k}-t_{k-1}>1, t_{k} \rightarrow \infty\right)$. Set

$$
\check{D}(A(s))=\left\{x \in \overline{D(A(s))}: L(s, x)=\lim _{h \rightarrow 0^{+}} h^{-1}|U(h+s, s) x-x|<\infty\right\} .
$$

Then $D(A(s)) \subset \check{D}(A(s)) \subset \overline{D(A(s))}$ and for $x \in D(A(s))$ we have

$$
|U(\bar{t}+s, s) x-U(t+s, s) x| \leq \omega^{-1}\left(e^{\omega \bar{t}}-e^{\omega t}\right)\left[L(s, x)+M_{T}\right], \quad \forall \omega>0
$$

(see [5, p. 25]). Since for $x \in D(A(s)), u(t)=U(t, s) x$ is a strong solution to (1.1) with $g(t) \equiv 0$ and $x_{0}=x$, we obtain

$$
\frac{1}{2} \frac{d}{d t}\left|u(t)-x_{0}\right|^{2}=\left(u^{\prime}(t), u(t)-x_{0}\right), \quad \text { a.e. } t \geq s .
$$

Analogously to Theorem 2.8 we can prove that there exists $\lim _{t \rightarrow \infty}\left|u(t)-x_{0}\right|$ for $x_{0} \in F$. Thus $h(t)=\left(u^{\prime}(t), u(t)-x_{0}\right) \in L(s,+\infty)$. We shall now prove that there exists a sequence $\tau_{j}$ such that $\tau_{j} \rightarrow \infty, h\left(\tau_{j}\right) \rightarrow 0$ and $U\left(\tau_{j}, s\right) x \stackrel{w}{\rightarrow} y$. For every $\epsilon>0(\epsilon<1 / 2)$ let $Q_{\epsilon}=\{t \geq s: h(t) \geq \epsilon\}$. The measure of $Q_{\epsilon}$ is finite since $h(t) \in L(s,+\infty)$ and therefore $Q_{\epsilon}$ can contain at most a finite number of the intervals $\left(t_{k}-\epsilon, t_{k}\right)$. It follows that there exists a $\tau$ large enough such that $h(\tau)<\epsilon$ and $0<t_{k}-\tau<\epsilon$ for some $t_{k}$ large enough. Therefore, we can choose a sequence $\tau_{j}$ such that $\tau_{j} \rightarrow \infty, 0<t_{k_{j}}-\tau_{j}<1 / j$ and $h\left(\tau_{j}\right)<1 / j$. By (2.18) we have

$$
\begin{gathered}
\left|U\left(t_{k_{j}}, s\right) x-U\left(\tau_{j}, s\right) x\right| \leq \frac{1}{j}\left(L(s, x)+M_{0}\right), \\
u\left(\tau_{j}\right)=U\left(\tau_{j}, s\right) x \stackrel{w}{\rightarrow} y .
\end{gathered}
$$

Since $u^{\prime}\left(\tau_{j}\right) \in A\left(\tau_{j}\right) u\left(\tau_{j}\right), \lim _{j \rightarrow \infty} h\left(\tau_{j}\right)=\left(u^{\prime}\left(\tau_{j}\right), u\left(\tau_{j}\right)-x_{0}\right)=0$. By the condition $\left(H_{3}\right)$ one obtains $y \in F$. The proof is complete.

Next, we shall consider the quasi-autonomous dissipative system

$$
\begin{cases}u^{\prime}(t) \in A u(t)+f(t), & t>0 \\ u(0)=x, & x \in \overline{D(A)}\end{cases}
$$

where $A$ is an $m$-dissipative operator and $f \in L(0, \infty ; H)$.

DEFINITION 2.11. A dissipative set $A$ is 3-dissipative if $\forall u_{1}, u_{2}, u_{3} \in D(A)$

$$
\left(A u_{1}, u_{1}-u_{2}\right)+\left(A u_{2}, u_{2}-u_{3}\right)+\left(A u_{3}, u_{3}-u_{1}\right) \leq 0 .
$$


THEOREM 2.12. Suppose $u(t)$ is an integral solution to (2.19), $F=A^{-1}(0) \neq \emptyset$. If $A$ is 3-dissipative, then for every $x \in \overline{D(A)}, \omega_{w}(x) \subset F$ and $u(t)$ is weakly convergent as $t \rightarrow \infty$

PROOF. Firstly, suppose $f(t)$ is of continuous bounded variation on $[0, T]$, $\bigvee_{0}^{T}(f)=M_{T} \leq M_{0}<\infty(\forall T>0)$, and there exists $T_{0}>s$ such that $f(t)=0$ for $t \geq T_{0}$. Set $A(t) x=A x+f(t)$ for all $x \in D(A)$ and $t \geq 0$. Then the equation (2.19) is equivalent to the evolution equation

$$
\begin{cases}u^{\prime}(t) \in A(t) u(t), & t>0 \\ u(0)=x, & x \in \overline{D(A(0))}=\overline{D(A)}\end{cases}
$$

where $A(t)$ is $m$-dissipative, $F=\bigcap_{t \geq T_{0}} A^{-1}(t)(0)=A^{-1}(0) \neq \emptyset$ and satisfies the conditions $\left(H_{1}\right)$ and $\left(H_{2}\right)$. Take $x_{0} \in F$, let $x_{n} \stackrel{w}{\rightarrow} x, y_{n} \in A\left(t_{n}\right) x_{n}=A x_{n}+$ $f\left(t_{n}\right)\left(t_{n} \rightarrow \infty\right)$ and $\left(y_{n}, x_{n}-x_{0}\right) \rightarrow 0$. By Definition 2.11, for $u \in D(A)$ and $v \in A u$ we have

$$
\begin{aligned}
0 & \geq\left(A x_{n}, x_{n}-x_{0}\right)+\left(A^{0} x_{0}, x_{0}-u\right)+\left(v, u-x_{n}\right) \\
& =\left(y_{n}, x_{n}-x_{0}\right)+\left(A^{0} x_{0}, x_{0}-u\right)+\left(v, u-x_{n}\right)-\left(f\left(t_{n}\right), x_{n}-x_{0}\right) .
\end{aligned}
$$

Letting $n \rightarrow \infty$, one obtains $(v, u-x) \leq 0, \forall[u, v] \in A$.

Thus $x \in F$ and the condition $\left(H_{3}\right)$ is valid. By Theorem 2.10, for every $x \in$ $\overline{D(A)}, \omega_{w}(x) \subset F$ and there is $w$ - $\lim _{t \rightarrow \infty} u(t)$.

For $f \in L(0, \infty ; H)$ there exists $f_{n} \in C_{0}^{\infty}(0, \infty ; H)$ such that $f_{n} \rightarrow f$ (in $L(0, \infty ; H))$. If $u_{n}(t)$ is an integral solution of an initial value problem

$$
\begin{cases}u_{n}^{\prime}(t) \in A u_{n}(t)+f_{n}(t), & t>0 \\ u_{n}(0)=x, & x \in \overline{D(A)}\end{cases}
$$

then clearly, there exists $s-\lim _{t \rightarrow \infty} u_{n}(t)=u(t)$ and the limit is uniformly convergent on $t \geq 0$. Moreover, by the proof above, for every $n$ there exists $w$ - $\lim _{t \rightarrow \infty} u_{n}(t)=p_{n}$. This implies that there exist $s-\lim _{t \rightarrow \infty} p_{n}=p$ and $w-\lim _{t \rightarrow \infty} u(t)=p$. The proof is complete.

REMARK 2.13. If $f(t) \equiv 0$ in Theorem 2.12, then for every $x \in \overline{D(A)}$ there exists $w-\lim _{t \rightarrow \infty} S(t) x$, where $S(t)$ is a non-linear contraction semigroup generated by $A$. This implies the conclusion of Proposition 2.14 in [7].

Let $-A=\partial \varphi$ be the subdifferential of an 1.s.c proper convex function. Then $A$ is a maximal 3-dissipative operator. Hence we get the conclusion of Theorem 2.3 in [4].

COROLlaRY 2.14. Let $-A=\partial \varphi$ be the subdifferential of an l.s.c proper convex function, $f \in L(0, \infty ; H)$ and $u(t)$ be an integral solution to (2.19). If $A^{-1}(0) \neq \emptyset$, then for every $x \in \overline{D(A)}$ there exists $w-\lim _{t \rightarrow \infty} u(t)$. 


\section{Examples}

Let $\Omega$ be a bounded domain in $\mathbb{R}^{n}$ with smooth boundary $\partial \Omega$ and $H=L^{2}(\Omega)$. Let $\beta \subset \mathbb{R}^{1} \times \mathbb{R}^{1}$ be a maximal monotone and $0 \in D(\beta)$. Then there exists an l.s.c proper convex function $j: \mathbb{R}^{1} \rightarrow(-\infty,+\infty]$ such that $\beta=\partial j$.

EXAMPLE 3.1. Consider the equation

$$
\begin{cases}\frac{\partial u}{\partial t} \in \Delta u-\beta(u(t, x))+f(t, x), & t>0, \text { a.e. } x \in \Omega \\ u(t, x)=0, & x \in \partial \Omega, t \geq 0 \\ u(0, x)=u_{0}(x), & \text { a.e. } x \in \Omega .\end{cases}
$$

Assume $0 \in R(\beta)$. For example

$$
\beta(x)= \begin{cases}{\left[-e^{-1}, e^{-1}\right]} & \text { if } x=0 \\ e^{-1}(1+x) & \text { if } x>0 \\ e^{-1}(x-1) & \text { if } x<0\end{cases}
$$

Then $\beta \subset \mathbb{R}^{1} \times \mathbb{R}^{1}$ is maximal monotone and $0 \in \beta(0)$. We set

$$
\varphi(u)= \begin{cases}2^{-1} \int_{\Omega}|\operatorname{grad} u|^{2} d x+\int_{\Omega} j(u) d x, & u \in H_{0}^{1}(\Omega), j(u) \in L(\Omega), \\ +\infty & \text { otherwise. }\end{cases}
$$

Then $\varphi: H \rightarrow(-\infty,+\infty]$ is an l.s.c proper convex function. The subdifferential

$$
\partial \varphi(u)=\left\{v \in L^{2}(\Omega): v(x) \in \beta(u(x))-\Delta u(x), \text { a.e. } x \in \Omega\right\}
$$

and $\partial \varphi^{-1}(0) \neq \emptyset$. If $u_{0} \in L^{2}(\Omega)$ and $f(t, x) \in L(0, \infty ; H)$, by Corollary 2.14 the integral solution $u(t)$ of the problem (3.1) is weakly convergent as $t \rightarrow \infty$ in $L^{2}(\Omega)$.

EXAMPLE 3.2. Let

$$
\beta(t) x=\left\{\begin{array}{ll}
{\left[-e^{-1}, e^{-1}\right],} & \text { if } x=0 \\
e^{-1}(1+x)+e^{-t} x, & \text { if } x>0 \\
e^{-1}(x-1)+e^{-t} x, & \text { if } x<0
\end{array} \quad \text { for } t \geq 0\right.
$$

Then $\beta(t)$ is a maximal monotone set in $\mathbb{R}^{1} \times \mathbb{R}^{1}$ for each $t \geq 0,0 \in D(\beta(t)), 0 \in$ $\beta(t)(0)$ for $t \geq 0$ and $D(\beta(t))=\mathbb{R}^{1}$ is independent of $t$. We consider the equation

$$
\begin{cases}\frac{\partial u}{\partial t} \in \Delta-\tilde{\beta}(t) u+g(t, x), & t \geq 0, \text { a.e. } x \in \Omega \\ u(t, x)=0 & x \in \partial \Omega, t \geq 0 \\ u(0, x)=u_{0}(x), & \text { a.e. } x \in \Omega\end{cases}
$$


where $g(t, x) \in L(0, \infty ; H)$ and $\tilde{\beta}(t)=\left\{[u, v]: u, v \in L^{2}(\Omega)\right.$ and $v(x) \in$ $\beta(t) u(x)$, a.e. $x \in \Omega$. Let

$$
D(A(t))=H^{2}(\Omega) \cap H_{0}^{1}(\Omega) \cap D(\tilde{\beta}(t)), \quad t \geq 0
$$

and

$$
A(t) u=\Delta u-\tilde{\beta}(t) u \quad \text { for } u \in D(A(t))
$$

Clearly, each $A(t)$ is $m$-dissipative in $H, D(A(t))=\mathscr{D}$ is independent of $t$ and $0 \in A^{-1}(t)(0)$ for all $t \geq 0$. Hence $\left(H_{2}\right)$ is satisfied. Further we can prove that $A(t)$ satisfies the condition $\left(H_{1}\right)$ and the conditions in Corollary 2.7 are valid. By Corollary 2.7, if $u_{0}(x) \in L^{2}(\Omega)$ and $u(t)$ is an integral solution of the problem (3.2), then $\sigma(t)$ is weakly convergent as $t \rightarrow \infty$ in $L^{2}(\Omega)$.

\section{References}

[1] V. Barbu, Nonlinear semigroups and differential equations in Banach spaces (Nordhoff, Groningen, 1976).

[2] H. Brésis, Operateurs maximaux monotones (North-Holland, Amsterdam, 1973).

[3] M. M. Israel Jr and S. Reich, 'Asymptotic behavior of solutions of a nonlinear evolution equation', J. Math. Anal. Appl. 83 (1981), 43-53.

[4] G. Morosanu, 'Asymptotic behaviour of solutions of differential equations associated to monotone operators', Nonlinear Anal. 3 (1979), 873-883.

[5] N. H. Pavel, Nonlinear evolution operators and semigroups, Lecture Notes in Math. 1260 (Springer, Berlin, 1987).

[6] A. Pazy, 'Strong convergence of semigroups of nonlinear contractions in Hilbert space', J. Analyse Math. 34 (1978), 1-35.

[7] —_, 'On the asymptotic behaviour of semigroups of nonlinear contractions in Hilbert space', J. Funct. Anal. 27 (1978), 292-307.

[8] S. Reich, 'Nonlinear evolution equations and nonlinear ergodic theorems', Nonlinear Anal. 1 (1977), 319-330.

[9] -, 'Almost convergence and nonlinear ergodic theorems', J. Approx. Theory 24 (1978), 269-272.

[10] B. D. Rouhani, 'Asymptotic behaviour of quasi-autonomous dissipative systems in Hilbert spaces', J. Math. Anal. Appl. 147 (1990), 465-476.

[11] Song Guozhu and Ma Jipu, 'Asymptotic behaviour of solutions to the nonlinear evolution equation', Sci. China Ser. A 23 (1993), 679-686.

\section{Department of Mathematics}

Nanjing University

Nanjing 210008

China 